\title{
Safety of Iron Fortification and Supplementation in Malaria-Endemic Areas
}

\author{
Gary M. Brittenham
}

Division of Pediatric Hematology, Department of Pediatrics, Columbia University, New York, NY, USA

\begin{abstract}
This review considers the safety of iron supplementation and fortification for the prevention and correction of iron deficiency in malaria-endemic areas, with a focus on potential means whereby provision of additional iron might heighten the risks of malaria and other infections. Iron deficiency itself may increase the risk of morbidity and mortality from malaria and other infections. The available evidence indicates that iron interventions are safe in settings without endemic malaria, and, with adequate health care, in regions with high transmission of malaria and other infections. Without regular surveillance and treatment of malaria and other infections, iron supplementation of individuals who are iron deficient seems safe, but individuals who are iron replete may have an increased risk of adverse outcomes. The mechanisms responsible for harmful effects with iron supplementation have not been established. These are likely to include the effects of (a) increased amounts of absorbed iron, with the production of plasma non-transferrin-bound iron, (b) increased amounts of iron in the gastrointestinal tract, with effects on gastrointestinal structural integrity and on gut microflora, and (c) the complex immune effects of iron interventions. Iron fortification appears to be generally safe, although more data from malaria-endemic areas are needed.
\end{abstract}

Copyright $\odot 2012$ S. Karger AG, Basel

\section{Introduction}

This review examines the safety of iron supplementation and fortification for the prevention and correction of iron deficiency in malaria-endemic areas. The focus is on potential means whereby provision of additional iron might heighten the risks of malaria and other infections. In settings without endemic 
malaria, studies have generally found no indication of iron-induced increases in infectious illness [1-5]. After summarizing the available evidence from prospective trials of iron interventions in malarial areas, proposed mechanisms of adverse interactions of iron interventions with malaria and other infections will be considered.

Iron supplements are iron preparations given orally to population groups for prevention and treatment of iron deficiency [6], typically without food in doses of 1-2 $\mathrm{mg} / \mathrm{kg}$ bodyweight. Iron fortification usually has the primary goal of preventing iron deficiency and includes both (a) the addition of iron to staple foods or condiments during processing and (b) the use of iron-containing powders, crushable tablets or spreads that are intended to be added to complementary foods prepared at home [6]. Iron-fortified processed foods and condiments provide lower levels of iron than supplements and are generally for adults, older children and adolescents. Iron preparations for home fortification supply intermediate levels of iron and are mainly for infants and young children. Iron fortification has generally been considered to be safe, but has not been specifically evaluated in malaria-endemic areas.

\section{Iron Interventions, Malaria and Other Infections}

Nearly half the world's population now lives in regions endemic for malaria in Africa, Asia and South America [7, 8]. In these same regions, iron deficiency, the most common form of malnutrition, further increases the risks of disability and death [9]. Lack of iron causes anemia, impairs cognitive and behavioral development, decreases work capacity, and when severe, increases mortality during pregnancy, infancy and childhood [10]. Iron deficiency and malaria usually are found together with other nutritional deficiencies and infectious pathogens, as well as with inherited red blood cell abnormalities, such as the thalassemias and sickle cell disorders. In these settings, iron nutrition can be improved by modifying the diet, by fortifying foods, treating helminth infections and by other public health interventions. Along with these measures, supplemental iron is needed for prevention and correction of iron deficiency, especially among infants, children and pregnant women [11].

Concerns about the safety of programs to provide additional iron have been long standing $[1,12]$. Earlier studies had generally suggested that the benefits of iron supplementation exceeded the risks $[2,13]$. In 2006, publication of the results of a large, randomized, controlled trial in Pemba, Zanzibar, Tanzania (hereafter, the Pemba trial) [14], was followed by a sustained reexamination of the risks of iron supplementation and fortification. Because of the importance of these results, the design and major findings of the Pemba trial will be outlined, together with subsequent appraisals of the safety of iron interventions in malaria-endemic regions by a World Health Organization (WHO) 
Consultation, by a systematic review from the Cochrane Collaboration, and by a Technical Working Group (TWG) convened by the Eunice Kennedy Shriver National Institute of Child Health and Human Development of the US National Institutes of Health and the Bill and Melinda Gates Foundation.

\section{Pemba Main Study}

The Pemba trial was a randomized, placebo-controlled trial of children aged 1-35 months living in Pemba, Zanzibar, Tanzania [14], in a setting where suboptimum care seeking and treatment in the routine public health system was associated with high mortality from infectious diseases. Children were assigned to daily oral supplementation with iron $(12.5 \mathrm{mg})$ and folic acid $(50 \mu \mathrm{g})$, iron, folic acid, and zinc, or placebo; children aged 1-11 months received half the dose. Most children were given supplements between meals. Based on available weights, the mean (SD) iron dose in $\mathrm{mg} / \mathrm{kg}$ per day for infants $0-5$ months old was $0.92(0.23)$, for infants $6-11$ months old was $0.80(0.14)$, and for children aged 12 months or older was $1.15(0.23)$. On the recommendation of the data and safety monitoring board, the iron and folic acid-containing groups of the trial were stopped early. At that time, 24,076 children contributed a follow-up of 25,524 child-years. Those who received iron and folic acid with or without zinc were $12 \%$ (95\% CI: $2-23 \%, \mathrm{p}=0.02)$ more likely to die or need treatment in hospital for an adverse event and 11\% $(1-23 \%, \mathrm{p}=0.03)$ more likely to be admitted to hospital; there were also $15 \%$ ( -7 to $41 \%$ ) more deaths in these groups, although this difference was not statistically significant $(\mathrm{p}=0.19)$. Overall, the analyses of cause-specific admissions to hospital and deaths in the main study indicated that malaria and other infectious diseases were significantly increased in children given iron and folic acid.

\section{Pemba Substudy}

The Pemba substudy included 2,413 children in whom baseline iron status and anthropometry were assessed. Although not an explicit aim, the children in the substudy were also provided with active surveillance for malaria and free treatment for infection in an attempt to balance the benefits and burdens of study participation $[4,15]$. In the children enrolled in the substudy, iron status at baseline seemed to modify the risk of adverse events. An elevated erythrocyte zinc protoporphyrin ( $\geq 80 \mu \mathrm{mol} / \mathrm{mol}$ heme) was used as the criterion for iron deficiency. As shown in figure 1, iron deficiency greatly increased the risk of severe illness and death. The rate of adverse events (hospitalizations and deaths) in iron-deficient children treated with placebo (9.76 events/100 child-years) was increased by $202 \%$ compared to that in iron-replete children (4.83 events/100 child-years; $\mathrm{p}=0.04)$. Iron and folate supplementation benefited iron-deficient children, reducing their rate of adverse events by $38 \%$ (to 6.00 events/100 childyears; $\mathrm{p}=0.02$ ). By contrast, iron and folate supplementation seemed to harm iron-replete children, increasing their risk of severe illness and death by $63 \%$ (to 


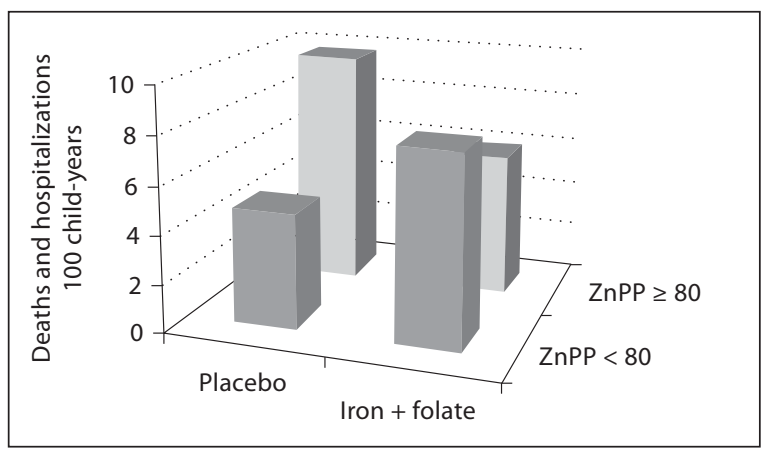

Fig. 1. Effects of supplementation with iron and folic acid on adverse events by iron status as assessed by erythrocyte zinc protoporphyrin (ZnPP) in the Pemba substudy among 2,413 children [14]. Iron and folate supplementation benefited iron-deficient $(\mathrm{ZnPP} \geq 80 \mu \mathrm{mol} / \mathrm{mol}$ heme) children but harmed iron-replete $(\mathrm{ZnPP}<80)$ children.

7.86 events/100 child-years), although this result was not statistically significant $(\mathrm{p}=0.24)$. Unlike the increase in adverse events observed in the main study, the overall effect of supplementation with iron and folic acid in the substudy was a non-significant reduction in adverse events. The different results between the main study and the substudy were interpreted as the result of more intensive diagnosis and management of children with malaria and other infections in the substudy. Although the increase in adverse events in iron-replete children given iron and folate in the substudy was not statistically significant, the investigators used this result to suggest that the increase in hospitalizations and deaths in the main study was the consequence of giving iron and folic acid supplementation to iron-replete children [14].

\section{WHO Consultation on Prevention and Control of Iron Deficiency in Infants and Young Children in Malaria-Endemic Areas}

After publication of the results of the Pemba trial, the WHO and the United Nations Children's Fund (UNICEF) issued a joint statement [16], and convened a WHO Consultation to examine the results of the Pemba trial. The WHO Consultation then made a series of recommendations:

'Universal iron supplementation for children under the age of 2 years is not recommended in malaria-endemic areas. However, iron therapy may have an important positive impact on child survival if it is directed to iron-deficient children in the setting of appropriate treatment of malaria and the complicating bacterial infections. Prior screening to identify iron-deficient children should be a necessary component of any such intervention' [6].

'The safety of iron preparations administered through home fortification of complementary foods for infants and young children, i.e. powders, crushable tablets, and fat-based spreads, is uncertain in malaria-endemic areas. Although there is reason 
to believe that those preparations may be safer than iron supplements, they cannot be recommended until this has been demonstrated' [6].

The WHO Consultation also noted that the safety of the use of processed foods fortified with iron in malarial areas had not been documented. Overall, because of the difficulties of diagnosing iron deficiency in resource-limited settings, the practical consequence of following these recommendations would be to halt programs of iron supplementation and fortification in malaria-endemic areas.

\section{Cochrane Review: Oral Iron Supplementation for Preventing or Treating Anemia} among Children in Malaria-Endemic Areas

A subsequent systematic review, published in 2009, examined additional data and information that were not included in the WHO Consultation [5]. The meta-analysis found that (a) the risk of clinical malaria was not increased by iron supplementation (RR: 1.00, 95\% CI: 0.88-1.13; 22,724 children, 14 trials) and (b) the risk was similar among children who were non-anemic (and thus less likely to be iron deficient) at baseline (RR: 0.96, 95\% CI: 0.85-1.09). Nonetheless, in trials that did not provide malaria surveillance and treatment, an increased risk of malaria with iron was observed (RR: 1.16, 95\% CI: $1.03-$ 1.31). Growth and other infections were generally not affected by iron supplementation. The conclusions of the review were that:

'Iron does not increase the risk of clinical malaria or death, when regular malaria surveillance and treatment services are provided. There is no need to screen for anæmia prior to iron supplementation' [5].

'Based on our review, routine iron supplementation should not be withheld for children living in malaria-endemic areas. Iron supplementation in malaria-endemic areas need not be based on baseline iron assessment; rather, emphasis should be on appropriate malaria prevention and surveillance' [5].

\section{National Institute of Child Health and Human Development /Gates Foundation Technical Working Group Review}

For regions with a high prevalence of malaria and other infections, the TWG evaluated potential mechanisms that might be responsible for adverse effects of iron, assessed the usefulness of biomarkers for assessing iron status, and reviewed the available evidence for the safety and effectiveness of iron supplementation and fortification [17], taking account of the results of the WHO Consultation and Cochrane Review. The TWG Iron and Malaria Technical Report [17] identified an overall consistency between the findings of the Pemba trial and the conclusions of previous reviews, the WHO Consultation, and the Cochrane review. All agreed that when iron supplementation is given in settings with inadequate health care services, the risk of malaria and other infections is increased. Iron supplementation with adequate medical surveillance and prompt treatment of malaria and other infections appeared to be safe. Altogether, the TWG noted the practical difficulties of determining the adequacy of health care and 
emphasized the need for further research to provide an improved understanding of the mechanisms underlying the adverse effects of iron interventions.

\section{Potential Mechanisms for Adverse Effects of Iron in Settings with a High Prevalence of Malaria and Other Infections and Inadequate Heath Care}

The adverse effects of iron observed in trials of iron supplementation almost certainly involve a complex interaction between iron status, the specific iron intervention, and malaria and other infections. Broadly, underlying potential mechanisms for adverse effects with iron supplementation or fortification may be considered as those resulting from (a) the increased amounts of iron absorbed, (b) the increased amounts of iron in the gastrointestinal tract, and (c) the immune effects of iron interventions. While a single mechanism might underlie the harmful effects of iron administration reported in the Pemba and other trials, a variety of mechanisms acting in concert is more likely to be responsible.

\section{Mechanisms for Adverse Effects of Iron Interventions Involving Increased Iron} Absorption

The WHO Consultation [6] and the TWG report [17] identified plasma nontransferrin-bound iron as a probable cause of the adverse events in the Pemba trial:

'When bolus doses of iron are administered parenterally or orally, especially without food, they may increase plasma iron concentrations and transferrin saturation and exceed the binding capacity of transferrin, leading to the appearance of non-transferrinbound iron (NTBI). NTBI is potentially toxic because it may promote free radical formation and be more readily available to pathogens' [6].

While direct toxic effects mediated by harmful free radical reactions $[18,19]$ could be involved, analyses of cause-specific admissions to hospital and deaths in the Pemba main study identified malaria and other infectious diseases as the most common causes of hospitalization and death in children given iron and folic acid [14].

For nonmalarial infections, the most likely explanation for an increased risk would seem to be plasma non-transferrin-bound iron being available to pathogens reaching the blood stream and enhancing their growth [20]. Concomitant bacteremia is common in patients with malaria infection. Bacterial virulence is greatly enhanced by freely available iron and the appearance of plasma non-transferrinbound iron could transform bacteremia into a serious or fatal infection.

With respect to the potential adverse effects of plasma non-transferrin-bound iron on malarial infection, direct donation of iron to Plasmodium falciparum seems unlikely. The available evidence indicates that the growth of $P$. falciparum in infected red blood cells is independent of host iron status [19]. Extracellular iron does not seem to be required for intra-erythrocytic parasite growth. Iron 
used by the parasite seems to be transported directly across the parasite plasma membrane from the infected red blood cell pool of cytosolic iron rather than from the digestive vacuole or from heme [21].

Plasma non-transferrin-bound iron has been reported to increase the expression of vascular endothelial adhesion molecules involved in sequestration of P. falciparum [22], providing a potential explanation for the more severe clinical course in children in the Pemba trial [14] given iron and folic acid. Increased sequestration of infected erythrocytes would increase the risk of more severe forms of malaria, especially of cerebral malaria, by worsening microvascular obstruction. Another potential mechanism would involve the hepatic phase of malarial infection [21]. Plasma non-transferrin-bound iron is rapidly removed from the portal circulation by hepatocytes. Increased iron availability within hepatocytes might enhance merozoite production, resulting in higher parasitemia during the erythrocytic phase of the infection.

To date, measurements of plasma non-transferrin-bound iron have not been reported in patients with malaria. In noninfected subjects, oral administration of doses of iron of about $1 \mathrm{mg} / \mathrm{kg}$ bodyweight, similar to those used in the Pemba trial, resulted in the appearance of plasma non-transferrin-bound iron in iron-deficient, anemic women [23] and in volunteers with normal iron stores [24]. Our own studies [25] in women with low iron stores (serum ferritin $<25 \mu \mathrm{g} / \mathrm{l}$ ), summarized graphically in figure 2 , have confirmed the appearance of plasma non-transferrin-bound iron after oral administration of ferrous sulfate, $60 \mathrm{mg}$ iron, or about $1 \mathrm{mg}$ iron $/ \mathrm{kg}$, similar to the dose of iron used in the Pemba trial. Giving the same dose of iron with a meal (rice with a vegetable sauce) greatly reduced the amounts of plasma non-transferrinbound iron formed. Giving a dose of iron of $6 \mathrm{mg} / \mathrm{kg}$, an amount comparable to that used in iron fortification, with the same meal failed to produce a significant increase in plasma non-transferrin-bound iron. Similar studies are needed in subjects in malaria-endemic areas, but these preliminary results suggest that oral iron supplements can produce plasma non-transferrinbound iron, that administration of iron supplements with food can decrease the amounts formed, and that lower doses, like those used in iron fortification, given with meals can further reduce or eliminate the production of plasma non-transferrin-bound iron.

\section{Mechanisms for Adverse Effects of Iron Interventions Involving Increased Amounts of Iron in the Gastrointestinal Tract}

Because only a fraction of supplementation and fortification iron is absorbed, most of the dose given passes into the lower small intestine and colon. Iron and iron status affect the structural and immunological integrity of the gastrointestinal tract as well as the gastrointestinal microflora, potentially promoting invasion by pathogenic enteric bacteria. Because $P$. falciparum sequesters within the gastrointestinal microvasculature, the inflammatory effects of malaria could 


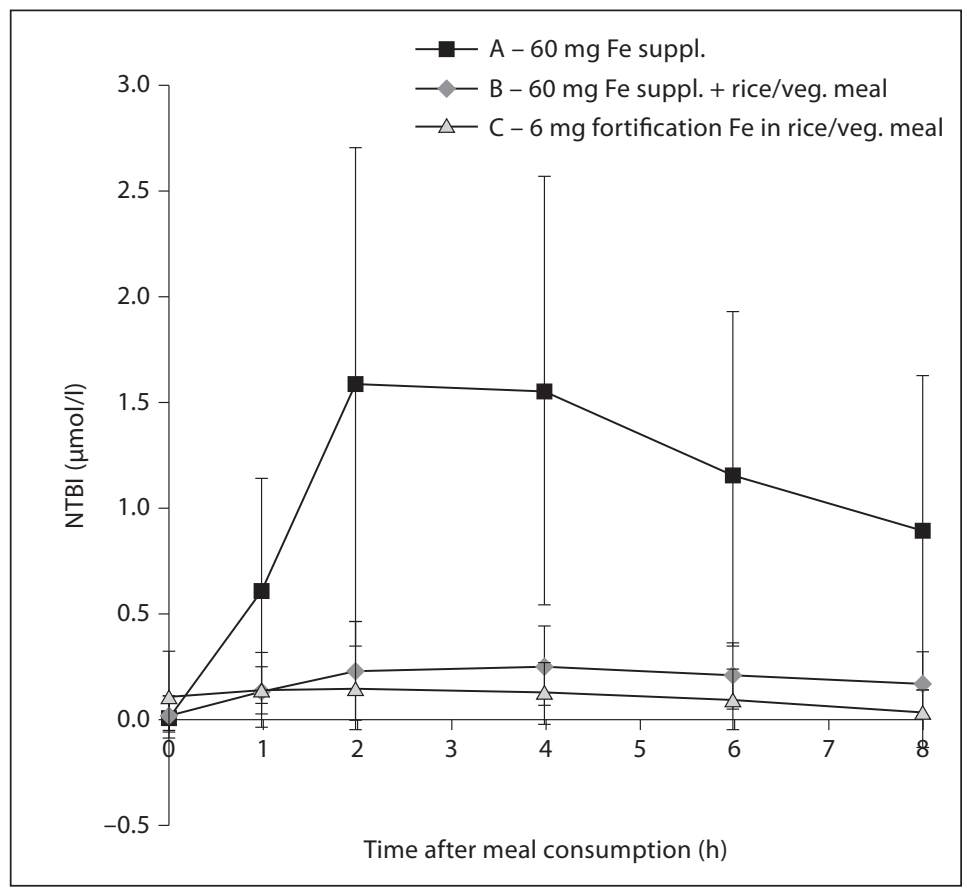

Fig. 2. Plasma non-transferrin-bound iron (NTBI) depends on the quantity of iron consumed and the presence of food [25]. In brief, 16 women with low iron stores (serum ferritin $<25 \mu \mathrm{g} / \mathrm{l}$ ) were administered $60 \mathrm{mg}$ iron as a ferrous sulphate solution, with and without a meal of rice and vegetable sauce, or the same meal without a supplement but containing $6 \mathrm{mg}$ iron as ferrous sulphate added as fortification iron to the meal. On combining results from the three test administrations, there was a strong correlation between increase in serum iron and the appearance of NTBI $\left(R^{2}=0.58\right)$.

interact with oxidant effects of iron on the gut wall, facilitating systemic bacterial invasion, bacteremia, and septicemia. Increased intestinal mucosal permeability has been reported in iron-supplemented Zambian children [26].

The normal gastrointestinal tract is populated by beneficial barrier bacteria that help protect against infection by preventing pathogenic colonization. Increases in gastrointestinal iron could alter this balance by enhancing the colonization and virulence of pathogenic enterobacteria [27]. Some studies have reported an increase in diarrhea in groups receiving iron supplements [2]. In a recent trial in African children, administration of iron-fortified biscuits resulted in a decrease in protective lactobacilli and an increase in fecal enterobacteria, together with an increase in mean fecal calprotectin, an indicator of gut inflammation [28]. In this population, iron fortification seemed to result in a more pathogenic gastrointestinal microbiota in association with evidence of increased gastrointestinal inflammation. 
Mechanisms for Adverse Effects of Iron Interventions Involving Immune Effects of Iron Interventions

Both iron and malaria modify host immune responses in complex and still poorly characterized fashions. An extensive cross-regulatory network interconnects iron metabolism and immune function. Iron alters cytokine secretion and transcription factors involved in the immune response, while both cellular and systemic iron homeostasis are modulated by acute-phase proteins and mediators derived from immune cells [29]. The withholding of iron from pathogens is a central component of host defense. Supplemental and fortification iron, by compromising iron withholding and interfering with the regulation and coordination of innate and adaptive immune defenses both systemically and within the gastrointestinal tract, might impair defenses against a variety of pathogens. In addition, malaria-induced dysregulation of innate and adaptive immune responses also interferes with protection against a variety of microorganisms [30].

\section{Conclusion}

Iron deficiency itself may increase the risk of morbidity and mortality from malaria and other infections. The available evidence indicates that efforts to prevent and control iron deficiency by iron supplementation and fortification are safe in settings without endemic malaria, and, with adequate health care, in regions with high transmission of malaria and other infections. Without regular surveillance and treatment of malaria and other infections, iron supplementation of individuals who are iron deficient seems safe, but individuals who are iron replete may have an increased risk of adverse outcomes. The mechanisms responsible for adverse effects with iron supplementation have not been established. These are likely to include the results of (a) increased amounts of iron absorbed, with the production of plasma non-transferrin-bound iron, (b) increased amounts of iron in the gastrointestinal tract, with effects on gastrointestinal structural integrity and on gut microflora, and (c) the complex immune effects of iron interventions. Iron fortification appears to be generally safe, although more data from malaria-endemic areas are needed. Further research is needed to provide an improved understanding of the mechanisms underlying the adverse effects of iron interventions.

\section{Acknowledgements}

Supported, in part, by grants from the US National Institutes of Health, including the Eunice Kennedy Shriver National Institute of Child Health and Human Development, the NIH Office of Dietary Supplements, the National Institute of Neurological Disorders and Stroke, the Fogarty International Center and from the Bill and Melinda Gates Foundation Gift Fund (U01 HD061233; R21 U01 HD064827; NS055348). 


\section{References}

1 Oppenheimer SJ: Iron and its relation to immunity and infectious disease. J Nutr 2001;131:616S-633S, discussion 633S-635S.

- Gera T, Sachdev HP: Effect of iron supplementation on incidence of infectious illness in children: systematic review. BMJ 2002;325:1142.

3 Tielsch JM, Khatry SK, Stoltzfus RJ, et al: Effect of routine prophylactic supplementation with iron and folic acid on preschool child mortality in southern Nepal: community-based, cluster-randomised, placebo-controlled trial. Lancet 2006;367: 144-152.

4 Stoltzfus RJ, Heidkamp R, Kenkel D, Habicht JP: Iron supplementation of young children: learning from the new evidence. Food Nutr Bull 2007;28:S572-S584.

5 Ojukwu JU, Okebe JU, Yahav D, Paul M: Oral iron supplementation for preventing or treating anaemia among children in malariaendemic areas. Cochrane Database Syst Rev 2009;CD006589.

-6 WHO Secretariat on behalf of the participants to the Consultation: Conclusions and recommendations of the WHO consultation on prevention and control of iron deficiency in infants and young children in malaria-endemic areas. Food Nutr Bull 2007; 28:S621-S627.

7 Hay SI, Guerra CA, Tatem AJ, et al: The global distribution and population at risk of malaria: past, present, and future. Lancet Infect Dis 2004;4:327-336.

8 Snow RW, Guerra CA, Noor AM, et al: The global distribution of clinical episodes of Plasmodium falciparum malaria. Nature 2005;434:214-217.

9 Stoltzfus RJ: Iron deficiency: global prevalence and consequences. Food Nutr Bull 2003;24:S99-S103.

10 Zimmermann MB, Hurrell RF: Nutritional iron deficiency. Lancet 2007;370:511-520.

11 Stoltzfus R: Defining iron-deficiency anemia in public health terms: a time for reflection. J Nutr 2001;131:565S-567S.

12 Murray MJ, Murray AB, Murray MB, Murray $\mathrm{CJ}$ : The adverse effect of iron repletion on the course of certain infections. BMJ 1978;2: 1113-1115.
13 INACG: Consensus Statement: Safety of Iron Supplementation Programs in MalariaEndemic Regions. Washington, ILSI, 1999.

14 Sazawal S, Black RE, Ramsan M, et al: Effects of routine prophylactic supplementation with iron and folic acid on admission to hospital and mortality in preschool children in a high malaria transmission setting: communitybased, randomised, placebo-controlled trial. Lancet 2006;367:133-143.

15 Stoltzfus R: Commentary: Cochrane Review on Oral iron supplementation for preventing or treating anaemia among children in malaria-endemic areas. Int J Epidemiol 2010; 39:34-35.

16 World Health Organization, UNICEF: Iron Supplementation of Young Children in Regions Where Malaria Transmission Is Intense and Infectious Disease Highly Prevalent. Geneva, World Health Organization 2006.

17 Raiten D, Namasté S, Brabin B: Considerations for the safe and effective use of iron interventions in areas of malaria burden. Int J Vitam Nutr Res 2011;81:57-71.

18 Esposito BP, Breuer W, Sirankapracha P, et al: Labile plasma iron in iron overload: redox activity and susceptibility to chelation. Blood 2003;102:2670-2677.

19 Hershko C: Mechanism of iron toxicity. Food Nutr Bull 2007;28:S500-S509.

20 Ratledge C: Iron metabolism and infection. Food Nutr Bull 2007;28:S515-S523.

21 Scholl PF, Tripathi AK, Sullivan DJ: Bioavailable iron and heme metabolism in Plasmodium falciparum. Curr Top Microbiol Immunol 2005;295:293-324.

-22 van Tits LJ, Jacobs EM, Swinkels DW, et al: Non-transferrin-bound iron is associated with plasma level of soluble intercellular adhesion molecule-1 but not with in vivo low-density lipoprotein oxidation. Atherosclerosis 2007;194:272-278.

23 Hutchinson C, Al-Ashgar W, Liu DY, et al: Oral ferrous sulphate leads to a marked increase in pro-oxidant nontransferrin-bound iron. Eur J Clin Invest 2004;34:782-784.

24 Dresow B, Petersen D, Fischer R, Nielsen $\mathrm{P}$ : Non-transferrin-bound iron in plasma following administration of oral iron drugs. Biometals 2008;21:273-276. 
25 Egli I, Brittenham GM, Forman JT, et al: The influence of supplementation and fortification iron doses on the formation of non-transferrin-bound iron (NTBI), unpublished, 2011.

26 Nchito M, Friis H, Michaelsen KF, et al: Iron supplementation increases small intestine permeability in primary schoolchildren in Lusaka, Zambia. Trans R Soc Trop Med Hyg 2006;100:791-794.

27 Raffatellu M, Baumler AJ: Salmonella's iron armor for battling the host and its microbiota. Gut Microbes 2010;1:70-72.
28 Zimmermann MB, Chassard C, Rohner F, et al: The effects of iron fortification on the gut microbiota in African children: a randomized controlled trial in Cote d'Ivoire. Am J Clin Nutr 2010;92:1406-1415.

29 Nairz M, Schroll A, Sonnweber T, Weiss G: The struggle for iron - a metal at the hostpathogen interface. Cell Microbiol 2010; 12:1691-1702.

30 Hawkes M, Li X, Crockett M, et al: Malaria exacerbates experimental mycobacterial infection in vitro and in vivo. Microbes Infect 2010;12:864-874. 